\title{
Some retarded nonlinear integral inequalities and their applications in retarded differential equations
}

Wu-Sheng Wang

Correspondence: wang4896@126. com

Department of Mathematics, Hechi University, Yizhou, Guangxi 546300, P. R. China

\section{Abstract}

In this article, we discuss some generalized retarded nonlinear integral inequalities, which not only include nonlinear compound function of unknown function but also include retard items, and give upper bound estimation of the unknown function by integral inequality technique. This estimation can be used as tool in the study of differential equations with the initial conditions.

2000 MSC: 26D10; 26D15; 26D20; 34A12; 34A40.

Keywords: integral inequality, integral inequality technique, Retarded differential equation, estimation

\section{Introduction}

Gronwall-Bellman inequalities [1,2] and their various generalizations can be used important tools in the study of existence, uniqueness, boundedness, stability, and other qualitative properties of solutions of differential equations, integral equations, and integral-differential equations.

Lemma 1 (Gronwall [1]). Let $u(t)$ be a continuous function defined on the interval [a, $a+h], a, h$ are nonnegative constants and

$$
0 \leq u(t) \leq \int_{a}^{t}[b u(s)+a] d s, \quad t \in[a, a+h]
$$

Then, $0 \leq u(t) \leq a h \exp (b h), \forall t \in[a, a+h]$.

Lemma 2 (Bellman [2]). Let $f, u \in C([0, h],[0, \infty)), h, c$ are positive constants. If $u$ satisfy the inequality

$$
u(t) \leq c+\int_{0}^{t} f(s) u(s) d s, \quad t \in[0, h] .
$$

Then, $u(t) \leq c \exp \left(\int_{0}^{t} f(s) d s\right), \quad t \in[0, h]$.

Lemma 3 (Lipovan [3]). Let $u, f \in C\left(\left[t_{0}, T\right), \mathbf{R}_{+}\right)$. Further, let $\alpha \in C^{1}\left(\left[t_{0}, T\right),\left[t_{0}, T\right)\right)$ be nondecreasing with $\alpha(t) \leq t$ on $\left[t_{0}, T\right)$, and let $c$ be a nonnegative constant. Then the inequality

(C) 2012 Wang; licensee Springer. This is an Open Access article distributed under the terms of the Creative Commons Attribution License (http://creativecommons.org/licenses/by/2.0), which permits unrestricted use, distribution, and reproduction in any medium, provided the original work is properly cited. 


$$
u(t) \leq c+\int_{\alpha\left(t_{0}\right)}^{\alpha(t)} f(s) u(s) d s, \quad t \in\left[t_{0}, T\right)
$$

implies that $u(t) \leq c \exp \left(\int_{\alpha\left(t_{0}\right)}^{\alpha(t)} f(s) d s\right), t \in\left[t_{0}, T\right)$.

Lemma 4 (Abdeldaim and Yakout [4]). We assume that $u(t)$ and $f(t)$ are nonnegative real-valued continuous functions defined on I and satisfy the inequality

$$
u(t) \leq u_{0}+\left(\int_{0}^{t} f(s) u(s) d s\right)^{2}+\int_{0}^{t} f(s) u(s)\left(u(s)+2 \int_{0}^{s} f(\tau) u(\tau) d \tau\right) d s, \forall t \in I_{1}(1.4)
$$

where $u_{0}$ be a positive constant. Then

$$
u(t) \leq u_{0} \exp \left(\int_{0}^{t} f(s) B_{1}(s) d s\right), \quad \forall t \in I,
$$

where

$$
B_{1}(t)=\frac{u_{0} \exp \left(4 \int_{0}^{t} f(s) d s\right)}{1-u_{0} \int_{0}^{t} f(s) \exp \left(4 \int_{0}^{s} f(\tau) d \tau\right) d s}, \forall t \in I,
$$

such that $u_{0} \int_{0}^{t} f(s) \exp \left(4 \int_{0}^{s} f(\tau) d \tau\right) d s<1$.

Lemma 5 (Abdeldaim and Yakout [4]). We assume that $u(t)$ and $f(t)$ are nonnegative real-valued continuous functions defined on I and satisfy the inequality

$$
u^{p+1}(t) \leq u_{0}+\left(\int_{0}^{t} f(s) u^{p}(s) d s\right)^{2}+2 \int_{0}^{t} f(s) u^{p}(s)\left(u(s)+\int_{0}^{s} f(\tau) u^{p}(\tau) d \tau\right) d s,(1.7)
$$

for all $t \in I$, where $u_{0}>0, p \in(0,1)$, are constants. Then

$$
u(t) \leq u_{0}^{\frac{1}{p+1}}+\frac{2}{p+1} \int_{0}^{t} f(s) B_{2}(s) d s, \quad \forall t \in I,
$$

where

$$
B_{2}(t)=\exp \left(\frac{2}{p+1} \int_{0}^{t} f(s) d s\right)\left(u_{0}^{\frac{1-p}{p+1}}+2(1-p) \int_{0}^{t} f(s) \exp \left(-2 \frac{1-p}{p+1} \int_{0}^{s} f(\tau) d \tau\right) d s\right)^{\frac{1}{1-p}},
$$

for all $t \in I$.

Lemma 6 (see [5]). Let $\phi \in C\left(\mathbf{R}_{+}, \mathbf{R}_{+}\right)$be a increasing function, $u, a, f \in C\left(\left[t_{0}, T\right), \mathbf{R}_{+}\right), a$ $(t)$ be a increasing function, and $\alpha \in C^{1}\left(\left[t_{0}, T\right),\left[t_{0}, T\right)\right)$ be nondecreasing with $\alpha(t) \leq t$ on $\left[t_{0}, T\right)$ where $T \in(0, \infty)$ is a constant. Then the inequality

$$
u(t) \leq a(t)+\int_{\alpha(0)}^{\alpha(t)} f(s) \varphi(u(s)) d s, \quad t \in\left[t_{0}, T\right)
$$


implies that

$$
u(t) \leq W^{-1}\left(W(a(t))+\int_{\alpha(0)}^{\alpha(t)} f(s) d s\right), \quad t \in\left[t_{0}, T_{1}\right),
$$

where

$$
W(t)=\int_{1}^{t} \frac{d t}{\varphi(t)} d s, \quad t>0,
$$

$W^{-1}$ is the reverse function of $W, T_{1}$ is the largest number such that

$$
W\left(a\left(T_{1}\right)\right)+\int_{\alpha(0)}^{\alpha\left(T_{1}\right)} f(s) d s \leq \int_{1}^{\infty} \frac{d t}{\varphi(t)} d s .
$$

There can be found a lot of generalizations of Gronwall-Bellman inequalities in various cases from literature (e.g., [3-13]).

In this article, we discuss some retarded nonlinear integral inequalities, where linear case $u(t)$ in integral functions in [4] is changed into the nonlinear case $\varphi(u(t))$, and the non-retarded case $t$ in [4] is changed into retarded case $\alpha(t)$, and give upper bound estimation of the unknown function by integral inequality technique.

\section{Main result}

In this section, we discuss some retarded integral inequalities of Gronwall-Bellman type. Throughout this article, let $I=[0, \infty)$.

Theorem 1. Let $\phi, \phi^{\prime}, \alpha \in C^{1}(I, I)$ be increasing functions with $\phi^{\prime}(t) \leq k, \alpha(t) \leq t, \alpha(0)$ $=0, \forall t \in I ; k, u_{0}$ be positive constants, we assume that $u(t)$ and $f(t)$ are nonnegative real-valued continuous functions defined on I and satisfy the inequality

$$
u(t) \leq u_{0}+\left(\int_{0}^{\alpha(t)} f(s) \varphi(u(s)) d s\right)^{2}+\int_{0}^{\alpha(t)} f(s) \varphi(u(s))\left(\varphi(u(s))+2 \int_{0}^{s} f(\tau) \varphi(u(\tau)) d \tau\right) d s,
$$

for all $t \in$ I. If $u_{0}^{-1}-k \int_{0}^{\alpha(t)} f(s) \exp \left(4 \int_{0}^{s} f(\tau) d \tau\right) d s>0$, then

$$
z(t) \leq \Phi^{-1}\left(\Phi\left(u_{0}\right)+\int_{0}^{\alpha(t)} f(s) B_{3}(s) d s\right), \forall t \in I
$$

where

$$
\begin{aligned}
& \Phi(x):=\int_{1}^{x} \frac{d s}{\varphi(s)}, \forall x>0 \\
& B_{3}(t):=\exp \left(4 \int_{0}^{\alpha(t)} f(s) d s\right)\left(\left(\varphi\left(u_{0}\right)\right)^{-1}-k \int_{0}^{\alpha(t)} f(s) \exp \left(4 \int_{0}^{s} f(\tau) d \tau\right) d s\right)^{-1} .
\end{aligned}
$$


Remark 1. If $\alpha(t)=t, \phi(u(s))=u(s)$, then Theorem 1 reduces Lemma 4 .

Proof. Let $z(t)$ denotes the function on the right-hand side of (2.1), which is a nonnegative and nondecreasing function on $I$ with $z(0)=u_{0}$. Then (2.1) is equivalent to

$$
u(t) \leq z(t), u(\alpha(t)) \leq z(\alpha(t)), \quad \forall t \in I .
$$

Differentiating $z(t)$ with respect to $t$, we have

$$
\begin{aligned}
\frac{d z}{d t}= & 2 \alpha^{\prime}(t) f(\alpha(t)) \varphi(u(\alpha(t))) \int_{0}^{\alpha(t)} f(s) \varphi(u(s)) d s+\alpha^{\prime}(t) f(\alpha(t)) \varphi(u(\alpha(t))) \\
& \times\left(\varphi(u(\alpha(t)))+2 \int_{0}^{\alpha(t)} f(\tau) \varphi(u(\tau)) d \tau\right) d s, \quad \forall t \in I .
\end{aligned}
$$

Using (2.5), we obtain

$$
\frac{d z}{d t} \leq \alpha^{\prime}(t) f(\alpha(t)) \varphi(z(\alpha(t))) w(t), \quad \forall t \in I,
$$

where $w(t):=\varphi(z(\alpha(t)))+4 \int_{0}^{\alpha(t)} f(s) \varphi(z(s)) d s, w(0)=\varphi(z(0))=\varphi\left(u_{0}\right), w$ is a nonnegative and nondecreasing function on $I$. By the monotonicity $\phi, \phi^{\prime}, z$, and $\alpha(t) \leq t$ we have $\phi(z(\alpha(t))) \leq w(t), \phi^{\prime}(z(\alpha(t))) \leq k$. Differentiating $w(t)$ with respect to $t$, and using (2.7) we have

$$
\begin{aligned}
\frac{d w}{d t} & \leq \varphi^{\prime}(z(\alpha(t))) \alpha^{\prime}(t) f(\alpha(t)) w^{2}(t)+4 \alpha^{\prime}(t) f(\alpha(t)) w(t) \\
& \leq k \alpha^{\prime}(t) f(\alpha(t)) w^{2}(t)+4 \alpha^{\prime}(t) f(\alpha(t)) w(t), \quad \forall t \in I
\end{aligned}
$$

By $w(t)>0$, we have

$$
w^{-2}(t) \frac{d w}{d t} \leq k \alpha^{\prime}(t) f(\alpha(t))+4 \alpha^{\prime}(t) f(\alpha(t)) w^{-1}(t), \quad \forall t \in I .
$$

Let $v(t)=w^{-1}(t)$, from (2.9) we have

$$
\frac{d v}{d t}+4 \alpha^{\prime}(t) f(\alpha(t)) v(t) \geq-k \alpha^{\prime}(t) f(\alpha(t)), \quad \forall t \in I .
$$

Consider ordinary differential equation

$$
\frac{d y}{d t}+4 \alpha^{\prime}(t) f(\alpha(t)) y(t)=-k \alpha^{\prime}(t) f(\alpha(t)), y(0)=\left(\varphi\left(u_{0}\right)\right)^{-1}, \quad \forall t \in I .
$$

The solution of Equation (2.11) is

$$
\begin{aligned}
y(t)= & \left(\varphi\left(u_{0}\right)\right)^{-1} \exp \left(-4 \int_{0}^{t} \alpha^{\prime}(s) f(\alpha(s)) d s\right) \\
& -\exp \left(-4 \int_{0}^{t} \alpha^{\prime}(s) f(\alpha(s)) d s\right) \int_{0}^{t} k \alpha^{\prime}(s) f(\alpha(s)) \exp \left(\int_{0}^{s} 4 \alpha^{\prime}(\tau) f(\alpha(\tau)) d \tau\right) d s \\
= & \left(\varphi\left(u_{0}\right)\right)^{-1} \exp \left(-4 \int_{0}^{\alpha(t)} f(s) d s\right)-\exp \left(-4 \int_{0}^{\alpha(t)} f(s) d s \int_{0}^{\alpha(t)} k f(s) \exp \left(4 \int_{0}^{s} f(\tau) d \tau\right) d s .\right. \\
= & \exp \left(-4 \int_{0}^{\alpha(t)} f(s) d s\right)\left(\left(\varphi\left(u_{0}\right)\right)^{-1}-k \int_{0}^{\alpha(t)} f(s) \exp \left(4 \int_{0}^{s} f(\tau) d \tau\right) d s\right) .
\end{aligned}
$$


By (2.10), (2.11), and (2.13), we obtain

$$
v(t) \geq \exp \left(-4 \int_{0}^{\alpha(t)} f(s) d s\right)\left(\left(\varphi\left(u_{0}\right)\right)^{-1}-k \int_{0}^{\alpha(t)} f(s) \exp \left(4 \int_{0}^{s} f(\tau) d \tau\right) d s\right)
$$

By the definition of $B_{3}(t)$ in (2.4) and the inequality (2.13), we have $w(t)<B_{3}(t), \forall t \in I$. From (2.7), we get

$$
\frac{d z}{d t} \leq \alpha^{\prime}(t) f(\alpha(t)) \varphi(z(\alpha(t))) B_{3}(\alpha(t)) \leq \alpha^{\prime}(t) f(\alpha(t)) B_{3}(\alpha(t)) \varphi(z(t)), \forall t \in I .
$$

By taking $t=s$ in the inequality (2.14) and integrating (2.14) from 0 to $t$, by the definition (2.3) of $\Phi$ we obtain

$$
z(t) \leq \Phi^{-1}\left(\Phi(z(0))+\int_{0}^{t} \alpha^{\prime}(s) f(\alpha(s)) B_{3}(\alpha(s)) d s\right) \leq \Phi^{-1}\left(\Phi(z(0))+\int_{0}^{\alpha(t)} f(s) B_{3}(s) d s\right),
$$

for all $t \in I$. This completes the proof of the Theorem 1 .

Theorem 2. Let $\psi(t), \phi(t), \phi(t) / t, \alpha(t) \in C^{1}(I, I)$ be increasing functions with $\psi^{\prime}(t)=\phi(t)$, $\alpha(t) \leq t, \alpha(0)=0, \forall t \in I ; k, u_{0}$ be positive constants, we assume that $u(t)$ and $f(t)$ are nonnegative real-valued continuous functions defined on I and satisfy the inequality

$$
\begin{aligned}
\psi(u(s)) \leq & u_{0}+\left(\int_{0}^{\alpha(t)} f(s) \varphi(u(s)) d s\right)^{2} \\
& +\int_{0}^{\alpha(t)} f(s) \varphi(u(s))\left(u(s)+2 \int_{0}^{s} f(\tau) \varphi(u(\tau)) d \tau\right) d s
\end{aligned}
$$

for all $t \in I$. Then

$$
u(t) \leq \exp \left(\Xi^{-1}\left(\Xi\left(\ln \left(1+\psi^{-1}\left(u_{0}\right)\right)+\int_{0}^{\alpha(t)} f(s) d s\right)+\int_{0}^{\alpha(t)} 4 f(s) d s\right)\right), \forall t \in\left(0, T_{2}\right) .
$$

where

$$
\Xi(t):=\int_{1}^{t} \frac{\exp (s) d s}{\varphi(\exp (s))}, \quad \forall t>0,
$$

$\Xi^{-1}, \psi^{-1}$ are the reverse function of $\Xi, \psi$ respectively, $T_{2}$ is the largest number such that

$$
\Xi\left(\ln \left(1+\psi^{-1}\left(u_{0}\right)\right)+\int_{0}^{\alpha(t)} f(s) d s\right)+\int_{0}^{\alpha(t)} 4 f(s) d s \leq \int_{1}^{\infty} \frac{\exp (s) d s}{\psi(\exp (s))}, \quad \forall x \in \mathbf{R}_{+} .
$$

Remark 2. If $\alpha(t)=t, \phi(u(t))=u^{p}(t), \psi(u(t))=u^{p+1}(t) /(p+1)$, by Theorem 2, we can obtain the result similar to Lemma 5 .

Proof. Let $\psi(z(t))$ denotes the function on the right-hand side of (2.16), then $z(t)$ is a nonnegative and nondecreasing function on $I$ with $z(0)=\psi^{-1}\left(u_{0}\right)$. Then $(2.16)$ is 
equivalent to

$$
u(t) \leq z(t), u(\alpha(t)) \leq z(\alpha(t)) \forall t \in I .
$$

Differentiating $\psi(z(t))$ with respect to $t$, we have

$$
\begin{aligned}
\psi^{\prime}(z(t)) \frac{d z}{d t}= & 2 \alpha^{\prime}(t) f(\alpha(t)) \varphi(u(\alpha(t))) \int_{0}^{\alpha(t)} f(s) \varphi(u(s)) d s+\alpha^{\prime}(t) f(\alpha(t)) \varphi(u(\alpha(t))) \\
& \times\left(u(\alpha(t))+2 \int_{0}^{\alpha(t)} f(\tau) \varphi(u(\tau)) d \tau\right) d s, \quad \forall t \in I .
\end{aligned}
$$

Using (2.19) and the relation $\psi^{\prime}(z(t))=\phi(z(t))$, from (2.20) we obtain

$$
\frac{d z}{d t} \leq \alpha^{\prime}(t) f(\alpha(t))\left(z(t)+4 \int_{0}^{\alpha(t)} f(s) \varphi(z(s)) d s\right), \quad \forall t \in I .
$$

Let $w(t):=z(t)+4 \int_{0}^{\alpha(t)} f(s) \varphi(z(s)) d s$, then $w(0)=z(0)=\psi^{-1}\left(u_{0}\right), z(t) \leq w(t), w$ is a nonnegative and nondecreasing function on $I$. Differentiating $w(t)$ with respect to $t$, and using (2.21) we have

$$
\begin{aligned}
\frac{d w}{d t} & \leq \alpha^{\prime}(t) f(\alpha(t)) w(t)+4 \alpha^{\prime}(t) f(\alpha(t)) \varphi(z(\alpha(t))) \\
& \leq \alpha^{\prime}(t) f(\alpha(t)) w(t)+4 \alpha^{\prime}(t) f(\alpha(t)) \varphi(w(\alpha(t))), \quad \forall t \in I .
\end{aligned}
$$

By $w(t)>0$, we have

$$
\frac{d w}{w(t) d t} \leq \alpha^{\prime}(t) f(\alpha(t))+4 \alpha^{\prime}(t) f(\alpha(t)) \varphi(w(\alpha(t))) / w(\alpha(t)), \quad \forall t \in I .
$$

Integrating (2.23) from 0 to $t$, we have

$$
\begin{aligned}
\ln w(t) & \leq \ln (1+w(0))+\int_{0}^{t} \alpha^{\prime}(s) f(\alpha(s)) d s+\int_{0}^{t} 4 \alpha^{\prime}(s) f(\alpha(s)) \varphi(w(\alpha(s)))(w(\alpha(s)))^{-1} d s \\
& \leq \ln (1+w(0))+\int_{0}^{\alpha(t)} f(s) d s+\int_{0}^{\alpha(t)} 4 f(s) \varphi(w(s))(w(s))^{-1} d s \\
& \leq \ln (1+w(0))+\int_{0}^{\alpha(t)} f(s) d s+\int_{0}^{\alpha(t)} 4 f(s) \varphi(\exp (\ln w(s)))(\exp (\ln w(s)))^{-1} d s,
\end{aligned}
$$

for all $t \in I$. Using Lemma 6 and the Definition (2.18) of $\Xi$, we obtain

$$
\begin{aligned}
\ln w(t) & \leq \Xi^{-1}\left(\Xi\left(\ln (1+w(0))+\int_{0}^{\alpha(t)} f(s) d s\right)+\int_{0}^{\alpha(t)} 4 f(s) d s\right) \\
& =\Xi^{-1}\left(\Xi\left(\ln \left(1+\psi^{-1}\left(u_{0}\right)\right)+\int_{0}^{\alpha(t)} f(s) d s\right)+\int_{0}^{\alpha(t)} 4 f(s) d s\right), \forall t \in\left(0, T_{2}\right) .
\end{aligned}
$$

Using the relation $u(t) \leq z(t) \leq w(t)$, we can obtain the estimation (2.17) of (2.16). 


\section{Application}

In this section, we apply our result to the following nonlinear differential equation [4]

$$
\left\{\begin{array}{l}
\frac{d x(t)}{d t}=F(t, x(\alpha(t)))+H(t, x(\alpha(t)), K(t, x(\alpha(t)))), \forall t \in I, \\
x(0)=x_{0}
\end{array}\right.
$$

where $x_{0}$ is a constant, $F, K \in C(I \times I, \mathbf{R}), H \in C\left(I^{3}, \mathbf{R}\right)$, satisfy the following conditions

$$
\begin{aligned}
& |F(t, x(\alpha(t)))| \leq f^{2}(\alpha(t))|\varphi(x(\alpha(t)))|^{2},|K(t, x(\alpha(t)))| \leq f(\alpha(t))|\varphi(x(\alpha(t)))| \\
& |H(t, x, y)| \leq|y|\left(\varphi(|x|)+2 \int_{0}^{t}|y| d s\right)
\end{aligned}
$$

where $f(t)$ is nonnegative real-valued continuous function defined on $I$.

Corollary 1. Consider nonlinear system (3.26) and suppose that $F, K, H$ satisfy the conditions (3.27) and (3.28). Let $\phi, \phi^{\prime}, \alpha \in C^{1}(I, I)$ be increasing functions with $\phi^{\prime}(t) \leq k$, $\alpha(t) \leq t, \alpha(0)=0, \forall t \in I, k$ be positive constants; then all solutions of Equation (3.26) exist on I and satisfy the following estimation

$$
|x(t)| \leq \Phi^{-1}\left(\Phi\left(\left|x_{0}\right|\right)+\int_{0}^{\alpha(t)} \frac{f(s)}{\alpha^{\prime}\left(\alpha^{-1}(s)\right)} B(s) d s\right), \forall t \in I,
$$

where

$$
\begin{aligned}
\Phi(x):= & \int_{1}^{x} \frac{d s}{\varphi(s)}, \forall x>0 \\
B(t):= & \exp \left(4 \int_{0}^{\alpha(t)} \frac{f(s)}{\alpha^{\prime}\left(\alpha^{-1}(s)\right)} d s\right) \\
& \times\left(\left(\varphi\left(\left|x_{0}\right|\right)\right)^{-1}-k \int_{0}^{\alpha(t)} \frac{f(s)}{\alpha^{\prime}\left(\alpha^{-1}(s)\right)} \exp \left(4 \int_{0}^{s} \frac{f(\tau)}{\alpha^{\prime}\left(\alpha^{-1}(\tau)\right)} d \tau\right) d s\right)^{-1} .
\end{aligned}
$$

Proof. Integrating both sides of the Equation (3.26) from 0 to $t$, we get

$$
x(t)=x_{0}+\int_{0}^{t} F(s, x(\alpha(s))) d s+\int_{0}^{t} H(s, x(\alpha(s)), K(s, x(\alpha(s)))) d s, \forall t \in I .
$$


From (3.27), (3.28), and (3.32) we obtain

$$
\begin{aligned}
|x(t)| \leq & \left|x_{0}\right|+\int_{0}^{t} f^{2}(\alpha(s))|\varphi(x(\alpha(t)))|^{2} d s \\
& +\int_{0}^{t} f(\alpha(s))|\varphi(x(\alpha(t)))|\left(\varphi(|x(\alpha(s))|)+2 \int_{0}^{s} f(\alpha(\tau))|\varphi(x(\alpha(\tau)))| d \tau\right) d s \\
\leq & \left|x_{0}\right|+\left(\int_{0}^{\alpha(t)} \frac{f(s)|\varphi(x(s))|}{\alpha^{\prime}\left(\alpha^{-1}(s)\right)} d s\right)^{2} \\
& +\int_{0}^{\alpha(t)} \frac{f(s)|\varphi(x(s))|}{\alpha^{\prime}\left(\alpha^{-1}(s)\right)}\left(\varphi(|x(\alpha(s))|)+2 \int_{0}^{s} \frac{f(\tau)|\varphi(x(\tau))|}{\alpha^{\prime}\left(\alpha^{-1}(\tau)\right)} d \tau\right) d s, \forall t \in I .
\end{aligned}
$$

Applying Theorem 1 to (3.33), we get the estimation (3.29). This completes the proof of the Corollary 1.

\section{Acknowledgements}

The authors are very grateful to the editor and the referees for their helpful comments and valuable suggestions. This research was supported by National Natural Science Foundation of China (Project No. 11161018), Guangxi Natural Science Foundation (Project No. 0991265), Scientific Research Foundation of the Education Department of Guangxi Province of China (Project No. 201106LX599), and the Key Discipline of Applied Mathematics of Hechi University of China (200725).

\section{Competing interests}

The author declares that they have no competing interests.

Received: 4 February 2012 Accepted: 29 March 2012 Published: 29 March 2012

\section{References}

1. Gronwall, TH: Note on the derivatives with respect to a parameter of the solutions of a system of differential equations. Ann Math. 20, 292-296 (1919). doi:10.2307/1967124

2. Bellman, R: The stability of solutions of linear differential equations. Duke Math J. 10, 643-647 (1943). doi:10.1215/S00127094-43-01059-2

3. Lipovan, O: A retarded Gronwall-like inequality and its applications. J Math Anal Appl. 252, 389-401 (2000). doi:10.1006/ jmaa.2000.7085

4. Abdeldaim, A, Yakout, M: On some new integral inequalities of Gronwall-Bellman-Pachpatte type. Appl Math Comput. 217, 7887-7899 (2011). doi:10.1016/j.amc.2011.02.093

5. Agarwal, RP, Deng, S, Zhang, W: Generalization of a retarded Gronwall-like inequality and its applications. Appl Math Comput. 165, 599-612 (2005). doi:10.1016/j.amc.2004.04.067

6. Bihari, IA: A generalization of a lemma of Bellman and its application to uniqueness problem of differential equation. Acta Math Acad Sci Hung. 7, 81-94 (1956). doi:10.1007/BF02022967

7. Pachpatte, BG: Inequalities for Differential and Integral Equations. Academic Press London (1998)

8. Kim, YH: On some new integral inequalities for functions in one and two variables. Acta Math Sinica. 21, 423-434 (2005). doi:10.1007/s10114-004-0463-7

9. Cheung, WS: Some new nonlinear inequalities and applications to boundary value problems. Nonlinear Anal. 64, 2112-2128 (2006). doi:10.1016/j.na.2005.08.009

10. Wang, WS: A generalized retarded Gronwall-like inequality in two variables and applications to BVP. Appl Math Comput. 191, 144-154 (2007). doi:10.1016/j.amc.2007.02.099

11. Wang, WS, Shen, C: On a generalized retarded integral inequality with two variables. J Inequal Appl 2008, 9 (2008). Article ID 518646

12. Wang, WS, Li, Z, Li, Y, Huang, Y: Nonlinear retarded integral inequalities with two variables and applications. J Inequal Appl 2010, 8 (2010). Article ID 240790

13. Wang, WS, Luo, RC, Li, Z: A new nonlinear retarded integral inequality and its application. J Inequal Appl 2010, 8 (2010). Article ID 462163

doi:10.1186/1029-242X-2012-75

Cite this article as: Wang: Some retarded nonlinear integral inequalities and their applications in retarded differential equations. Journal of Inequalities and Applications 2012 2012:75. 